\title{
Daycase Bariatric Surgery-Just How Much Experience Is Potentially Lost to Trainees?
}

\author{
B. S. Grewal • A. M. El-Sharkawy • D. L. J. Morris • \\ J. W. Quarmby • T. E. Rowlands
}

Published online: 25 June 2013

(C) Springer Science+Business Media New York 2013

Dear Sir,

Recent changes prompted by the 2003 European Working Time Directive (EWTD) have impacted surgical training in a number of ways. Although the EWTD has decreased trainee fatigue and improved work-life balance, it has led to decreased theatre exposure for surgical trainees. The knock-on effect has been to reduce trainee opportunities to learn and consolidate their surgical technique. Bariatric surgery is complex, requiring a high level of skill with a laparoscopic approach at the heart of such operations. In order to ensure competence, trainees need to utilise all available opportunities to learn and perform parts or indeed all of the operation appropriate to their grade and ability. We reviewed electronic database records detailing daycase operations between 1 January 2007 and 1 January 2012 at a large NHS foundation trust, in order to investigate the availability of opportunities for trainees to develop laparoscopic skills in bariatric pro- cedures. There were 92 daycase bariatric procedures performed during this time, including gastric band portreplacement and laparoscopic band-adjustment procedures. All these cases were performed by consultants with trainees assisting in only $20 \%(n=18)$ of cases. Possible reasons for poor trainee participation include trainees not wishing to utilise such an opportunity or trainees being timetabled to attend other activities. Relatively minor operations such as daycase bariatric procedures provide trainees with opportunity to develop a solid technical foundation from which to progress to more complex procedures in the future. The laparoscopic skills developed by performing these subspecialty procedures are core and transferable to other surgical specialties. In order for standards of surgical excellence within the UK to be maintained, trainees must utilise all such available opportunities.

B. S. Grewal $(\bowtie) \cdot$ A. M. El-Sharkawy • D. L. J. Morris •

J. W. Quarmby • T. E. Rowlands

Royal Derby Hospital, Derby, UK

e-mail: ballyg88@gmail.com 